\title{
Depresión en niños y niñas con cáncer
}

\author{
Yolanda del Refugio González Hernández \\ Facultad de Psicología, Universidad Autónoma de San Luis Potosí, México
}

Dirección postal: Amado Nervo № 130. Barrio de Tesquisquiapan. CP 788230.

Ce: yolgon00@hotmail.com

\begin{abstract}
Resumen. La depresión infantil existe y está presente en muchos niños y niñas hoy en día, principalmente porque se han incrementando los factores de riesgo para su aparición. Uno de estos grandes factores es la pérdida de la salud, la amenaza de daño a la propia estructura física, psicológica y social del niño o la niña. El cáncer infantil logra romper esta estructura. La presente revisión bibliográfica pretende exponer aquellos estudios que se han realizado sobre la depresión infantil, su relación con el cáncer, así como el análisis de los resultados de estudios longitudinales de sobrevivientes de cáncer infantil sobre la presencia o ausencia de secuelas psicosociales importantes que influyan en su funcionamiento actual.
\end{abstract}

Palabras clave: depresión infantil, cáncer, ajuste psicológico.

Abstract. Infant depression exists and is present in many children nowadays, mainly because of the increment of the risky factors for their appearance. One of those several factors are the lost of health, and the threat of damage to the own children's physic, psychological and social structures. The present bibliographic review intends to expose those studies that have been done about infant depression, its relation with cancer, as well as the analysis of the results of the longitudinal studies about survivors of infant cancer upon the presence or absence of important psychosocial sequels that influence on their actual functioning.

Key Words: Infant depression, cancer, psychological adjustment. 


\section{Introducción}

La existencia o no de la depresión infantil como entidad clínica ha sido un tema que durante décadas ha ocupado el estudio de la comunidad científica. Esto se debe principalmente al hecho de que el área de salud mental infantil durante un largo tiempo no fue considerada de gran relevancia. Hoy en día resulta imposible siquiera intentar negar su existencia. La depresión en niños, niñas y adolescentes se está manifestando en forma considerable. El avance en la tecnología, las demandas sociales y los ritmos de vida hacen que el mundo gire mas rápidamente y con ello sus exigencias. $\mathrm{El}$ niño/a se ve envuelto/a en una vida llena de compromisos, demandas, competencias y en su mismo proceso evolutivo debe adquirir las habilidades y destrezas que le permitan sobrevivir y aún más sobresalir.

Los niños y las niñas experimentan emociones desde los primeros días de vida y las perciben en los demás entre los 6 y 9 meses llegando a ser concientes de las propias alrededor de los 5 años. La psicología evolutiva considera que a partir del año y medio de edad los niños y niñas son capaces de tener un control sobre sus propias emociones y este es el punto para adaptarse a las normas sociales en las que está siendo educado (Del Barrio y Moreno, 1996; Del Barrio, 2000 a).

Para Deuber (1982), la depresión existe y puede manifestarse en los niños y las niñas desde los seis años de edad. Del Barrio (2000 b) afirma "...también los niños se deprimen ya que tienen las mismas emociones que los adultos pero con sus propias características: son más frecuentes, más intensas, más versátiles y menos reflexivas". Pauchard (2002), argumenta que los/as niños/as y adolescentes pueden sufrir de depresión al igual que los adultos, la alteración se presenta en muchas formas con grados y duración variados. Considera que los niños y las niñas que viven bajo mucha tensión, que han experimentado una pérdida importante o que tienen desórdenes de la atención, del aprendizaje o de conducta corren mayor riesgo de sufrir depresión. La propia enfermedad puede ser considerada como un factor de riesgo ya que los niños y las niñas experimentan una pérdida importante en este caso de su propia salud.

El estudio formal de la depresión infantil se remonta hacia los años cuarenta con estudios de Akerson, Spitz, Bowlby (Del Barrio, 2000 a) quienes mediante la observación directa del comportamiento infantil encontraron conductas que hoy son reconocidas como típicas de la depresión. Conforme se avanza en el estudio de la depresión y de la internalización de los procesos se acepta la existencia de este trastorno como concepto y entidad psicopatológica primero por el Instituto Nacional de Salud Mental de Estados Unidos de Norteamérica en 1977, y en 1980 el DSM-III admite la existencia de la depresión infantil y con ello se abren las puertas para su 
estudio y abordaje terapéutico (Del Barrio, 2000 a; Nissen, 1987). A partir de 1990 se localizan y aíslan los factores de riesgo que pueden llevar a la presencia de depresión infantil, así como el análisis de los síntomas, la eficacia de los tratamientos psicofarmacológicos y los estudios de seguimiento. Una parte fundamental en el abordaje de este tema es la creación, aplicación y adaptación de los instrumentos de medición propios para esta entidad clínica (Del Barrio, 2001).

En el caso del cáncer infantil es necesario conocer aquellos aspectos que al surgir la enfermedad se hacen evidentes y afectan psicológicamente en los niños y las niñas así como en sus familiares. Considerando que uno de los factores de riesgo para la existencia de la depresión infantil es la presencia de una enfermedad médica, se hace en este artículo una revisión bibliográfica, buscando establecer la presencia o no de depresión en aquellos infantes que han sufrido de cáncer y determinar las posibles secuelas psicológicas que se han encontrado en estos menores ahora ya adultos jóvenes. Se considera que uno de los principales obstáculos para su detección temprana es el poder separar con límites claros la depresión como tal y la depresión derivada de las propias condiciones físicas, del uso de fármacos, o de cualquier otro tratamiento propio de esta enfermedad tales como la radiación o quimioterapia.

\section{Depresión infantil}

Los expertos en el diagnóstico de la depresión infantil enfatizan la importancia de entender la depresión como una entidad distinta de la depresión en los adultos y esto se basa principalmente en el hecho de que el/la niño/a se encuentra en un proceso de desarrollo lo que no ocurre con el adulto (Del Barrio, 2000 a; Malmquist, 1983; Woffe, Grier y Klar, 2000).

La depresión es un trastorno afectivo en el cual se encuentran implicados aspectos orgánicos, emocionales, cognitivos, motores y sociales (Del Barrio, 2001). Para Del Barrio (1997) la depresión infantil es un cambio permanente de la capacidad de disfrutar los acontecimientos, de comunicarse con los demás y un cambio en el rendimiento escolar y esto va acompañado de acciones que pueden ser consideradas como conductas de protesta o de rebeldía. Los porcentajes de depresión infantil detectados entre la población en general pueden estimarse entre un 8\% y 10\% (Del Barrio V., 2000 b).

La presencia de depresión infantil es determinada cuando existe una situación afectiva de tristeza de gran intensidad y duración. Esta puede ser entendida como depresión mayor cuando los síntomas prevalecen por más de dos semanas o bien como un trastorno distímico cuando la ocurrencia de estos síntomas pasan de un mes. Los expertos en el tema después de numerosas investigaciones han establecido la presencia de síntomas y signos 
específicos de esta entidad clínica (Del Barrio 1997; DSM IV) tales como: tristeza, irritabilidad, anhedonia (pérdida de placer), llanto fácil, falta de sentido del humor, sentimiento de no ser querido, baja autoestima, aislamiento social, cambios en el sueño, modificación en la conducta alimenticia y peso, hiperactividad, disforia y/o ideas suicidas.

En la depresión infantil, el término puede referirse a un síntoma, a un síndrome, a un conjunto de respuestas psicológicas o a una enfermedad. La duración e intensidad de la manifestación de la conducta serán los síntomas diferenciales del desorden, así por ejemplo, la tristeza de un niño o niña puede responder a un trauma y esta será entonces de corta duración a diferencia de si la depresión permanece por largo tiempo y va asociada con insomnio, irritabilidad, cambios en los hábitos alimenticios, alteraciones en la escuela y en el ámbito social que será entonces diagnosticada como una enfermedad. La depresión no se refiere a un estado transitorio de tristeza sino a un desorden que afecta principalmente el potencial mismo del niño o niña. En algunos casos la clínica de la depresión infantil se presenta como irritabilidad, dificultad para concentrarse y atender. Algunos síntomas que acompañan a la depresión en niños y niñas escolarizados/as son anorexia, letargo, tristeza, llanto, agresión, hiperactividad, somatización, temor a la muerte, frustración, desesperanza, baja autoestima y auto-critica, dificultades de aprendizaje, fallos en el procesamiento visual que impiden el acceso a la lectura, lentitud en sus movimientos, hostilidad hacia padres y maestros, pérdida de placer en aquellas actividades que previamente le satisfacían (Del Barrio 1997, 2000 a; Clay, 1999; Deuber, 1982). En el caso del diagnóstico de la depresión en niños y niñas es relevante considerar su situación familiar, su nivel de maduración emocional así como sus habilidades para enfrentar la enfermedad y su tratamiento, su edad y su nivel de desarrollo así como su experiencia previa con enfermedades y su fortaleza personal (Archenbach, 1983).

Generalmente los síntomas de la depresión infantil tienen un carácter exteriorizado y de protesta lo que hace difícil su reconocimiento, así se pueden encontrar trastornos de conducta, rasgos de ansiedad, fallos escolares, etc., que encubren la sintomatología de la depresión haciendo difícil en la mayoría de los casos su diagnóstico (Del Barrio, 2000 a). Una historia familiar de enfermedad mental, de abusos físicos o sexuales, la pérdida de seres queridos, la separación de los padres, el desarraigo social, un cambio de domicilio o ciudad, pueden hundir en muchas ocasiones a los más pequeños en la depresión, que afirma Fresneda (2003) es la cuarta enfermedad más extendida hoy en día.

Son varios los factores de riesgo predisponentes para la existencia de la depresión infantil. Estos factores pueden ser de "tipo personal" como son el temperamento y la personalidad; los infantes introvertidos tienen mayor tendencia a la depresión que los extrovertidos. La autoeficacia, la baja 
autoestima, las atribuciones negativas, las expectativas, también juegan un papel importante en los factores de riesgo personales cuando estas no se cubren o por el contrario rebasan los límites de control del sujeto. También se deben de considerar los "factores físicos", es decir, la existencia de bases biológicas de la depresión, el factor hereditario. Otros factores de riesgo son de "carácter social" tales como la familia, la estabilidad y la afectividad, si hay carencia de éstos para el/la niño/a éste/a puede tender a la depresión. Un factor más es la clase social a la que pertenece el niño o la niña o incluso al país en donde vive, lo cual se debe principalmente a las exigencias de vida en los países desarrollados. También se habla de "acontecimientos vitales negativos" como el fracaso escolar, la ruptura de amistades, la muerte o enfermedad de un familiar, la propia enfermedad, accidentes, etc. Todos ellos pueden o no estar presentes en un menor deprimido, sin embargo, por si mismos son factores de riesgo a considerar (Del Barrio, 2000 a, 2000 b) y es precisamente el factor de la propia enfermedad, el cáncer, el punto de abordaje para esta revisión.

\section{Depresión y cáncer}

En el mundo actual es importante conocer las enfermedades que con el tiempo se han ido incrementando y presentando con mayor frecuencia debido al aumento de demandas de los patrones y estilos de vida actuales, afectando el desarrollo y la rutina de las personas. La depresión es una condición médica-afectiva seria que afecta pensamientos, sentimientos y la habilidad para funcionar en la vida diaria que puede presentarse a cualquier edad.

En pacientes con enfermedades físicas la depresión puede estar asociada accidentalmente, ser una complicación de la propia enfermedad física o bien causar o exacerbar síntomas somáticos tales como fatiga, malestar o dolor. El termino depresión describe un espectro de alteraciones de la afectividad que pueden ir de moderadas a severas o bien de transitorias a persistentes. Los síntomas depresivos son continuamente observados en cualquier población pero deben de ser considerados clínicamente cuando estos síntomas interfieren con actividades de la vida diaria y persisten por más de dos semanas. En éste caso el diagnóstico de enfermedad o desorden depresivo puede realizarse. El diagnóstico depende principalmente de la presencia de dos síntomas cardinales: disminución persistente del humor o afectividad y pérdida de interés o placer en actividades usuales (Peveler, Carson y Rodin, 2002).

La depresión se considera un síndrome que afecta aproximadamente al $15 \%$ o $25 \%$ de los pacientes en general a quienes se les diagnostica el cáncer y a sus familiares (Derogatis, Morrow y Fettings, 1983; Henriksson, Isometsä 
y Hietanen, 1995). Los pacientes con cáncer sufren de una variedad de síntomas, Donnelly y Walsh (1995) y Bottomley (1998), han reportado dolor, fatiga y anorexia entre los más comunes. El dolor, la anorexia, la ansiedad, pérdida de energía, fatiga severa, saciedad temprana, constipación, tienden a ser los síntomas moderados o severos (Naughton y Homsi, 2002). Cuando a alguien se le diagnostica esta enfermedad debe enfrentarse a una serie de emociones y situaciones estresantes muy difíciles de manejar tales como: considerar la posibilidad de una muerte, ver truncados los planes de vida, cambios en la imagen corporal y el autoconcepto, cambios en el rol social y estilo de vida. Es por ello que resulta fácil comprender que aparezca la depresión en cualquier persona quién se enfrenta a este diagnóstico y en quiénes lo rodean.

Se ha confirmado que el diagnóstico y tratamiento de la depresión puede dar una oportunidad para impactar sobre la mortalidad como también en la calidad de vida (Augelino y Treisman, 2001; Petersen y Quintivan, 2002). Una parte crítica del enfrentamiento del cáncer es el reconocimiento de los niveles de depresión presentes (Block, 2000; McDaniel, Musselman, Porter, Reed y Nemeroff, 1995), así como el uso de criterios claramente definidos para el diagnóstico de la depresión en pacientes con cáncer estableciendo una base para su estudio e implementación de intervenciones terapéuticas específicas (Bukberg, Penman y Holland, 1984).

Algunas de los principales causas para la depresión en gente que padece cáncer son: los factores de riesgo relacionados directamente con la enfermedad tales como, la depresión en el tiempo del diagnóstico, pobre control del dolor, avance en el estado del cáncer, estresores en la vida, incremento de alteraciones físicas, tipo de cáncer así como el tratamiento con ciertos agentes de quimioterapia. También existen factores de riesgo que no se relacionan directamente con la enfermedad: historia personal de depresión, pérdida de soporte familiar, historia familiar de depresión o suicidio, historia de alcoholismo o abuso de drogas, problemas psicológicos previos y tratamiento psicoterapéutico, entre otros (Henriksson, et al. 1995; Lloyd Williams y Friedman, 2001; Lynch, 1995; Massie y Holland, 1987; Meyer y Mark, 1995).

Sin embargo, también existen muchos mitos acerca del cáncer y cómo la gente se enfrenta a él. Uno de los principales es que las personas con cáncer están siempre deprimidas, se considera que la depresión es normal en las personas que sufren de cáncer, o bien que los tratamientos son inútiles ya que cada una de estas personas tendrá un constante sufrimiento y una muerte dolorosa. Se piensa que la tristeza y la culpa son sentimientos propios de quien padece el cáncer. Ciertamente toda la gente experimentará estas reacciones en algún o algunos periodos de la enfermedad, pero no tienen que convertirse en una constante por lo que es importante poder hacer una distinción entre grados normales de tristeza y desórdenes de depresión. 
Aunque hasta cierto punto es claro que la valoración de los síntomas en pacientes con cáncer no es generalmente la más correcta, cierta población especial corre mayor riesgo de ser valorada inadecuadamente. Esta población incluye a ancianos, menores e individuos con alteraciones cognitivas (Naughton y Homsi, 2002).

\section{La presencia de depresión en niños y niñas con cáncer}

El diagnóstico de la depresión en niños enfermos y niñas enfermas es difícil debido a que algunos síntomas físicos son usualmente considerados como manifestaciones de un desorden depresivo que puede ser resultado de la alteración médica por si misma. Las consecuencias de la depresión en niños y niñas enfermos/as incluye tanto incapacidad como morbosidad, las cuales se asocian con la depresión en cualquier paciente. Las investigaciones producen una amplia evidencia según la cual los niños y las niñas que sufren de enfermedades médicas crónicas o inhabilitantes incrementan el riesgo de presentar desajustes psicológicos y psicosociales. Algunos padres y profesionales de la salud pueden creer que los menores son incapaces de sentir dolor, sin embargo, hoy en día se sabe que aún los niños y niñas más pequeños/as saben qué es el dolor y pueden describirlo como un sentimiento sobre una gran variedad de dimensiones (Watters y Williamson, 1999).

Los niños enfermos y las niñas enfermas que se encuentran deprimidos/as constituyen un grupo especial de alto riesgo quiénes pueden sufrir consecuencias severas mucho más allá de las esperadas en otra población médica (Masi, Favilla, Mucci y Millepiedi, 2000; Shemesh, Bartell, y Newcorn, 2002). De hecho, uno de cada diez infantes experimenta una alteración crónica alrededor de los 15 años. La prevalencia de la depresión entre muestras de niños, niñas y adolescentes de la población en general, es decir, menores sanos, es similar para hombres y mujeres hasta la adolescencia. En este tiempo, la sintomatología depresiva se incrementa y se mantiene en la población femenina. Al mismo tiempo, diferencias de género en cuadros depresivos no han sido encontradas en niños y niñas jóvenes o adolescentes con alteraciones médicas (Essau y Petermann, 1995; Petersen, Compas, Brooks-Gunn, Stemmler, y Grant, 1993). Los desórdenes depresivos son una causa común de alteración en adultos y menores (Birmaher y Brent, 1998; Druss, Rosenbeck y Sledge, 2000), sin embargo, la depresión no se presenta necesariamente en todos los pacientes clínicamente enfermos (Bennett, 1994).

Un diagnóstico rápido y oportuno así como el tratamiento de la depresión en personas médicamente enfermas es importante, sobre todo porque la depresión cuando se presenta se agudiza debido a la incapacidad 
que provoca la enfermedad médica. Se ha mostrado repetidamente que está asociada con un pobre resultado médico y/o la muerte tanto en menores como adultos quiénes tienen que vivir con un tratamiento médico de por vida. Valorar por separado los síntomas que se relacionan con la depresión de aquellos que no, es una tarea muy complicada la cual idealmente deberá hacerse por un especialista en el área quién debe estar familiarizado no sólo con los criterios de la depresión infantil sino también con las manifestaciones clínicas de la enfermedad y su tratamiento, por ejemplo, la fatiga se espera que aparezca durante una fase de la quimioterapia y éste síntoma entonces no pertenece a un cuadro primario de depresión (Shemesh, et al., 2002).

Por otro lado, una cuestión a considerar en la valoración de la depresión infantil es la necesidad de contar con múltiples informantes para su diagnóstico. Así, se tiene por un lado la información del padre y de la madre, de los propios niños y niñas, compañeros y compañeras y en ocasiones de los maestros y maestras, y por lo general se reportan grandes discrepancias entre estos informes. Esta diferencia se agudiza en el grupo de niños y niñas con enfermedades médicas ya que en ocasiones el padre y la madre de estos niños y niñas pueden no distinguir con claridad los síntomas o alteraciones del estado de ánimo de sus hijos e hijas de aquellos síntomas considerados como propios de la enfermedad que padecen (Canning, Hanser, Shade y Boyce, 1992; Mulhern, Fairclough, Smith y Douglas, 1992; Woffe, et al., 2000). Finalmente no se puede dejar de lado la cuestión del tratamiento, ya que los patrones de respuesta infantil y los cambios que van constantemente experimentando con respecto a la enfermedad médica, hacen aún más complicada la distinción de la depresión como entidad clínica independiente.

Los niños y las niñas con cáncer pueden sufrir algunos síntomas similares en frecuencia a la de los pacientes adultos con cáncer (Naughton y Homsi, 2002) pero esto no es suficiente para considerarlas y estudiarlas como una misma entidad. Cuando a un niño o a una niña se le diagnóstica cáncer pueden ser muchos los pensamientos y sentimientos que puede experimentar lo cual le dificulta la comprensión inmediata de lo que está sucediendo. Cuando este infante es conciente de la gravedad de la enfermedad hay mayor ansiedad la cual aumenta con el progreso de la enfermedad y con cada visita al médico u hospital. Aún los niños y niñas a quienes nunca se les ha comunicado su diagnóstico se dan cuenta de la ansiedad que existe entre las personas que les rodean. Para algunos especialistas esta falta de información puede producir mayor tensión en el menor y ser el origen de fantasías perturbadoras. Aunque los niños y las niñas en remisión indican que han vencido la amenaza de la muerte, parece no desaparecer la ansiedad asociada a ella. Esta incertidumbre crónica sobre la recaída y sobrevivencia es la que plantea una amenaza importante para la vida del niño o niña con cáncer. 
Niños y niñas pueden sentirse cohibidos o incapaces de realizar cierta clase de actividades debido a su delicado estado de salud. Es así porque tienen que acudir constantemente al hospital para su tratamiento y seguir estrictamente regímenes alimenticios que de una $u$ otra forman generan cierta insatisfacción. Esto tiene como resultado cuestionamientos sobre cada situación de él o ella comparada con la de otras personas o infantes. El malestar físico, el dolor, la debilidad, etc. causado por el tratamiento son una complicación importante ("Depresión PDQ", 2003).

Gran cantidad de niños y niñas se sienten relativamente bien en el momento del diagnóstico, pero en el momento del tratamiento éste puede parecer peor que la enfermedad. El tratamiento del cáncer conlleva un fuerte conflicto debido a la ansiedad y al dolor asociados a procedimientos médicos, las náuseas y vómitos por la quimioterapia. Todo esto puede manifestarse con diferentes síntomas como pesadillas, insomnio, depresión, anorexia y retraimiento. También debido al tratamiento niños y niñas pueden experimentar una serie de cambios en su cuerpo que pueden ser reversibles, como la pérdida o ganancia de peso, pérdida de pelo, úlceras bucales o bien en el peor de los casos secuelas permanentes como amputación, esterilidad, daño cerebral, secuelas cognitivas, etc. El cambio en la imagen física es un recordatorio constante para niños y niñas de ser diferente a los demás pudiendo afectar su autoconfianza, lo cual puede llevar a un comportamiento agresivo, retraimiento y miedo a ir a la escuela entre otras consecuencias. Por otra parte el aislamiento que suponen las hospitalizaciones puede conducir a problemas psicosociales, en la mayoría de las ocasiones debido al alto grado de implicación de los padres y del personal médico, especialmente con niños y niñas pequeños/as o bien a la restricción de la actividad en el niño o niña.

Son varias las hipótesis que pretenden explicar el aumento de riesgo de padecer depresión en el niño o niña enfermo/a. Se pueden mencionar el elevado número de días de hospitalización, la severidad de los síntomas físicos, los síntomas de depresión de la madre, la afectación de la autoimagen y el autoconcepto, los efectos a largo plazo del tratamiento tales como una capacidad reducida de atención, problemas de aprendizaje e infertilidad entre otros (Bennett, 1994; Bottomley, 1998; Bukberg, et al., 1984; Ressel, 2003; Shemesh, et al., 2002).

La mayoría de niños y niñas que se enfrentan al diagnóstico de cáncer han demostrado, emocionalmente, una adecuada adaptación a esta situación así como en su desarrollo y un positivo crecimiento psicosocial. Una minoría de este grupo sin embargo, desarrollan problemas psicológicos, incluida la depresión, ansiedad, trastornos del sueño, dificultades en las relaciones interpersonales y no cooperación con el tratamiento siendo necesaria su referencia a un especialista de salud mental (Kazak y Meadows, 1989; Links y Stockwell, 1985; Noll, Bukowski, LeRoy, Rogosch y Kulkarni, 1991). 
Bennett (1994) realizó un estudio de síntomas depresivos entre niños/as y adolescentes con problemas médicos crónicos. Los resultados indican un elevado factor de riesgo de presentar síntomas depresivos entre niños y niñas con problemas médicos moderados y severos aunque concluye que existe un mayor riesgo en algunas entidades clínicas tales como el asma, dolor abdominal recurrente, más que en pacientes con cáncer, fibrosis quística, o diabetes mellitus. Sin embargo, el tiempo del diagnóstico, la edad y el género, no fueron estudiados ni relacionados con los síntomas.

En algunos de los primeros estudios realizados sobre depresión en niños y niñas con cáncer no se han encontrado datos suficientes que avalen una relación significativa entre ellos. Los resultados arrojan niveles promedio de depresión similares a los de la población en general. Investigaciones posteriores han observado altos niveles de depresión y ansiedad en niños/as y adolescentes con cáncer en comparación con grupos de niños/as sin cáncer (Kashani y Hakami, 1982). Estudios subsecuentes demuestran bajos niveles de depresión en infantes en tratamiento del cáncer comparados con un grupo control sano (Worchel, Nolan, Willson, Purser, Copelan \& Pfefferbaum, 1988), así como decremento de ansiedad y depresión en adolescentes, tres semanas después de haber recibido el diagnóstico de cáncer o un adecuado manejo de depresión, ansiedad y autoconcepto en niños y niñas en tratamiento para el cáncer en comparación a sus iguales de control (Allen, Newman y Souhani, 1997; Canning, et al., 1992; Nerville, 1996; Noll, Gaartstein, Vannatta, Correll, y William, 1999; Worchel, et al., 1988).

Von, Enskär, Kreuger y Sjödén (2000) hacen una revisión de investigaciones que muestran algunos factores de riesgo relacionados con dificultades psicosociales en niños/as y adolescentes durante y al término del tratamiento de cáncer. Los resultados indican que en una población de niños y niñas entre 9 y 18 años durante el tratamiento presentaron altos niveles de ansiedad en comparación con quienes ya habían terminado el tratamiento (Barley Moore y Mosher, 1997). Pero otros autores (Frank, Blount y Brown, 1997) han demostrado que los niños y niñas en tratamiento no difieren significativamente de aquellos que lo han finalizado con respecto a niveles de depresión y ansiedad. Haciendo una revisión sobre los ajustes de los niños y niñas con cáncer en un tiempo mayor, Eiser y Havermans (en Von, et al., 2000) concluyeron que mientras más temprano se realiza el diagnóstico se incrementa la vulnerabilidad psicológica. En contraparte Koocher, O’Malley, Gogan, y Foster (1980) consideran que un diagnóstico en etapas posteriores es un factor de riesgo para desarrollar dificultades psicosociales.

Una serie de estudios avala los resultados según los cuales no existen diferencias significativas entre infantes con cáncer e infantes sin cáncer en las medidas de ansiedad, depresión o el auto-concepto. Sin embargo, con respecto a los resultados de estas investigaciones se mencionan diferencias 
significativas en el tamaño de las muestras, el procedimiento en los tratamientos, el diagnóstico, el tiempo de valoración y los instrumentos, entre otros puntos, impidiendo así la generalización de los resultados (Moe y Holen, 2000; Von, et al., 2000). Según Sean (2002) para muchos investigadores, los niños y las niñas con cáncer u otras enfermedades crónicas frecuentemente se adaptan a su condición reprimiendo sus emociones y enfrentándose a sus sentimientos de depresión y ansiedad. Pero estos resultados muestran un alto nivel de defensa y una tendencia a la negación de su estado de tristeza, sin llegarse aún a establecer si esto ayuda o no realmente al niño y la niña.

También se han estudiado otros factores de riesgo tales como la relación entre niveles altos de depresión y su relación con la etapa del desarrollo en que se realiza el diagnóstico, así como las secuelas físicas o bien la relación entre un largo tiempo para la administración del tratamiento, recaídas múltiples y problemas psicosociales. Von, et al., (2000), realizaron un estudio cuyo propósito era estimar los niveles de auto-concepto, depresión y ansiedad entre niños/as y adolescentes suizos con edades entre 8 y 18 años en o finalizado el tratamiento de cáncer y los resultados comparados con un grupo de pares de niños/as suizos/as sin alteración médica. Los resultados no mostraron diferencias entre estos grupos con respecto a los factores estudiados apoyando así los resultados de estudios anteriores. Otros estudios han reportado que los niños y niñas con cáncer se sienten mal con respecto a su apariencia física y su imagen corporal en comparación con el grupo sano, y esto influye directa o indirectamente en la presencia de rasgos depresivos. Los autores concluyen que la apariencia física es un aspecto del autoconcepto, afecta a los niños y las niñas con cáncer y es ahí donde la intervención psicosocial debe enfocarse.

Un grupo de investigaciones ha llegado a concluir que en el periodo posterior al tratamiento, hay un incremento de riesgo de fallos en las funciones psicosociales mayor al periodo del tratamiento (Kaplan, Busner, Weinhold y Lenon, 1987; Mulhern et al., 1992 y Ouvinen-Birgerstam citado en Von, et al., 2000).

Para Grau y Cañete (2000), es importante considerar la etiología del cáncer. Así el niño o la niña con tumor intracraneal puede presentar alteraciones emocionales y cambios comportamentales producidos no sólo por el aumento de la presión craneal y la localización del tumor, sino también por la situación de estrés que es vivida por los niños/as y su familia como consecuencia de las hospitalizaciones y tratamientos. Algunos de los trastornos del comportamiento más frecuentes son: impaciencia e irritabilidad; apatía, indiferencia ante el entorno y depresión; perturbaciones emocionales como impulsividad, suspicacia, euforia y desinhibición, fatiga, dependencia hacia los adultos y falta de cooperación. Las alteraciones emocionales y del carácter tienen una influencia negativa en la experiencia y 
ajuste social. Estas dificultades se manifiestan en un pobre autocontrol, baja autoestima, aislamiento social, insatisfacción respecto a las relaciones con sus compañeros y falta de capacidad para tener amigos. Es importante considerar los instrumentos de valoración de los síntomas en éstos niños y niñas sobre todo durante situaciones de dolor para mejorar la unificación de criterios (Lebaron y Zeltzer, 1984, Naughton, et al., 2002).

Balen, Fielding y Lewis (1998), presentaron los hallazgos de una investigación aplicado con un grupo de pacientes pediátricos británicos con actividades para una semana. El estudio mostró el incremento de independencia, protección y habilidades entre los padres y los infantes de este grupo, mejorando con ello la actitud de los padres hacia la propia enfermedad y por ende entre niños y niñas. Las investigaciones y propuestas de intervención están dirigiendo sus pasos hacia el abordaje de las secuelas reconocidas del cáncer, tanto a nivel neurocognitivo como psicosocial y a los factores claramente identificados como factores de riesgo, para el desarrollo posterior de alteraciones en estas áreas.

La mayoría de los niños y las niñas pueden lidiar con el caos emocional ocasionado por el cáncer, y dar buenas muestras de adaptación, crecimiento y desarrollo psicosocial positivo. Sin embargo, una minoría de ellos/as desarrolla problemas psicológicos tales como depresión, ansiedad, trastornos del sueño y dificultad en las relaciones interpersonales, evitando en muchos casos continuar con el tratamiento establecido.

Con base en la revisión precedente se puede considerar la depresión por si misma, menos relacionada con el diagnóstico del cáncer y más relacionada con la inconformidad de una enfermedad crónica, el efecto de la enfermedad sobre la apariencia física, las relaciones con los compañeros y el desempeño en la escuela así como el miedo constante de la posible presencia de recaídas. Es decir, infantes con cáncer viven bajo una amenaza constante de vida, involucrados en tratamientos invasivos y dolorosos predisponiendo a presentar dificultades emocionales y psicosociales.

\section{Estudios de sobrevivientes de cáncer y secuelas psicológicas}

El tratamiento exitoso de infantes con cáncer ha incrementado su tasa de sobrevivencia en comparación con infantes cuyo diagnóstico de cáncer se hizo 30 años atrás. Estos avances médicos han llevado a dirigir la atención hacia las consecuencias psicosociales que pudieran presentarse en estos adultos jóvenes sobrevivientes. El estudio de las secuelas en pacientes oncológicos se ha enfatizado mucho y estas secuelas han sido relacionadas con el tratamiento aplicado así como el tipo de neoplasia presentada. Se consideran sobrevivientes a largo plazo aquellas personas en remisión 
completa 5 años después de establecerse el diagnóstico o dos años después de finalizar el tratamiento. Se cree que existen importantes secuelas psicológicas y sociales en estas personas, sin embargo, aún a la luz de una extensa investigación no se ha podido llegar a una conclusión única o determinante.

El abordaje de las secuelas psicológicas se ha hecho realizando una diferenciación entre aspectos cognitivos y emocionales. Las secuelas neurocogntivas del cáncer infantil se han centrado en la neurotoxicidad de la radioterapia craneal y la quimioterapia, intratecal y sistémica, el efecto de la dosis y la edad de irradiación, la relación entre el tipo de alteraciones neurocognitivas y las alteraciones detectadas por la neuroimagen. Dependiendo de la etiología del cáncer se han subdividido los estudios y se han analizado los resultados con relación al CI total. Con respecto al ámbito emocional, los estudios se han centrado en la presencia de conductas ansiosas y/o depresivas, considerando que el diagnóstico del cáncer puede ocasionar grados elevados de estrés que se traducen en la aparición de estos síntomas.

Otros problemas específicos abordados son las dificultades académicas, la preocupación sobre la fertilidad e insatisfacción en las relaciones interpersonales significativas, la excesiva dependencia de los padres, el decremento en el interés en las relaciones sociales, los problemas emocionales y conductuales, la alteración de la actitud hacia la sexualidad y la excesiva utilización de la negación como mecanismo de defensa (Benedito, López, Serra, Harto, Gisbert, Mulas, y Freís, 2000; White y Macleod, 2002).

Los primeros estudios de sobrevivientes infantiles de cáncer, demuestran un adecuado desarrollo con un funcionamiento dentro de los límites normales en donde la depresión no aparece como un rasgo significativo. Algunos hallazgos de estos estudios también han enfatizado que las reacciones de ansiedad son más comunes entre la población de pacientes jóvenes mientras que la depresión es más característica de la población adulta (Deuber, 1982; Fritz, Williams y Amylon, 1988; Greenberg, Kashani y Hakami, 1989; Kaplan, et al., 1987; Kazak y Meadows, 1989; Kazak, 1989; Koocher, et al., 1980; Tebbi, Bromberg y Mallon, 1988).

Por lo general se considera que a menor edad es más fácil observar retraimiento, ansiedad, depresión así como menor grado de actividad y competencia social en el momento del diagnóstico y estas alteraciones se van atenuando conforme avanza el tiempo (Benedito, et al., 2000; Sawyer, Antoniou, Nigoyen, Tooogood y Rice, 1995), considerando que la presencia de depresión no llega a ser fuertemente significativa entre éstos pacientes con cáncer. Estudios realizados con infantes sobrevivientes al cáncer por Koocher, et al. (1980), manifiestan que cerca de la mitad del grupo presentaron síntomas psiquiátricos tales como depresión, ansiedad y problemas de autoestima. Bauld, Anderson, y Arnold, (1998) por su parte 
reportaron que infantes sobrevivientes de cáncer con edad entre 12 y 17 años no muestran diferencias significativas con respecto a niveles de ansiedad o auto-concepto en comparación con el grupo de pares sin cáncer.

En 1999, Noll, et al. en su estudio sobre el funcionamiento social, emocional y conductual de infantes con cáncer hipotetizaron que los niños y las niñas con cáncer podrían tener más problemas sociales y dificultades con el ajuste emocional que sus pares sin cáncer. Estudiaron a una muestra de niños y niñas con edades entre los 8 y los 15 años, valorando relaciones interpersonales con sus compañeros, ajuste emocional y conductual a través de reportes de los padres, maestros/as, sus compañeros/as y autoreportes. Los resultados refieren que con relación al grupo control, los niños y las niñas con cáncer fueron percibidos por sus maestros/as como menos sociables, por maestros/as y compañeros/as como menos agresivos/as y con gran aceptación social por parte de sus pares. Con respecto a las medidas de depresión, ansiedad, soledad, y auto-concepto no hubo diferencias significativas excepto en los niños y las niñas con cáncer que mostraron menor satisfacción con respecto a las competencias atléticas debido a la limitación de sus actividades. Tampoco hubo discrepancia en la percepción del padre o de la madre con respecto a problemas de conducta, emocionales o de funcionamiento social. Concluyen así pues, los niños y las niñas con cáncer quienes habían recibido quimioterapia obtuvieron un puntaje igual al de sus pares y aun mejor en algunas áreas de funcionamiento social.

Para Benedito et al. (2000) la mayoría de los pacientes oncológicos se enfrentan de manera adecuada al cáncer. Un $25 \%$ de ellos puede necesitar un apoyo psicológico que le permita abordar la sintomatología ansiosa o depresiva presente en algún período de la enfermedad. Los factores de riesgo son el distanciamiento o la alteración de las relaciones interpersonales, la dependencia de los demás, la incapacidad para desarrollar tareas de la vida cotidiana, los cambios en la imagen corporal y el cuestionamiento de aspectos existenciales. Consideran que el tiempo para la adaptación a la enfermedad oscila entre tres meses y dos años, transcurrido este tiempo las alteraciones psiquiátricas graves deben ser una excepción.

Links et al. (1985) afirman la evidencia de secuelas psicológicas, neuropsicológicas y sociales en infantes sobrevivientes al cáncer y mencionan la necesidad de trabajar en la prevención de éstas secuelas.

Por su lado Teta, et al. (citado en Benedito, et al., 2000) no encontraron una mayor prevalencia de depresión, intento de suicidio, ni ingresos en centros psiquiátricos en los sobrevivientes de cáncer pediátrico comparados con sus hermanos y hermanas. Los y las adolescentes con cáncer tienden a pelear por sobrevivir más que a sucumbir a la muerte o decidirse por el suicidio. Para la mayoría de los/as jóvenes el suicidio responde más a una crisis y no tanto a un desorden mental (Perrone, 1993; Stein, Witztum, y DeNour, 1989). 
Rechner (1990), realizó un estudio fenomenológico diseñado para tratar de entender y describir la experiencia de adolescentes con cáncer. Se trabajó con adolescentes quiénes habían recibido o estaban en tratamiento para el cáncer, se audio-grabaron las entrevistas y después fueron analizados los temas de interés. Los resultados muestran que en términos generales, los adolescentes tienen la meta de ganarle a la vida, responden al cáncer experimentando la enfermedad y considerando que ellos fueron normales, permitiéndoles desarrollar una filosofía de pensamientos positivos, redefiniendo su mundo social en orden a sobrevivir. Con base en ello, los autores presentan propuestas para un adiestramiento en afrontar la vida con base en lo espiritual, ayudando a niños y sobrevivientes con cáncer a salir adelante y superar la depresión, ansiedad y mejorar su calidad de vida (Boeving, 2000; Watson, Haviland, Greer, Davidson y Bliss, 1999). El rango de depresión mayor entre adolescentes con cáncer no es más grande que el de la población en general, sin embargo, se concluye que estos datos no excluyen la posibilidad de síntomas enmascarados los cuales sólo bajo ciertas condiciones pueden llegar a ser claramente obvios (Tebbi, et al., 1988).

Existen estudios según los cuales los jóvenes con cáncer quienes han recibido constantemente quimioterapia presentan un alto riesgo de sufrir dificultades psicosociales principalmente en dos áreas: el ajuste social con sus compañeros/as y en el adecuado nivel emocional (Noll, et al., 1991; Bennett, 1984). Watson, et al. (2002) investigaron la calidad de vida en jóvenes sobrevivientes del cáncer. Los resultados indican que estos/as jóvenes consideran los síntomas asociados con el tratamiento con tendencias a desaparecer y son mínimos. Sin embargo, otros efectos permanecen por más tiempo tales como la fatiga, los dolores, y la negación del dolor impactando sobre la calidad de vida. La mayoría de ellos puntúan alto en escalas como felicidad, sentirse útiles, satisfacción en la vida así como su habilidad de afrontar el cáncer, pero su esperanza esta marcada por la incertidumbre. Por otro lado refieren que las actividades espirituales y religiosas proporcionan un propósito de vida y los cambios se perciben positivamente como resultado de asociar el cáncer con una adecuada calidad de vida. Ello da lugar a considerar la importancia de manejar cambios en las estructuras del pensamiento no como una técnica sino como un estilo de vida, un idioma en particular que permita cambios internos e igualmente el manejo de esperanza, intereses y motivos (Ringdal, 1995; Watson, et al., 1999; Wilkinson y Kitzinger, 2000).

Sawyer, Antoniou, Tooogood \& Rice (1997) siguieron prospectivamente la adaptación de jóvenes, padres y familiares durante los dos primeros años posteriores al diagnóstico de cáncer. Para ello se contó con una muestra de niños y niñas con diagnóstico de cáncer en edades comprendidas entre los dos y cinco años, así como con sus padres y familiares. Se realizaron tres valoraciones, una inmediata al diagnóstico, al 
año y a los dos años después del diagnóstico. En cada una de las valoraciones se contó con un grupo control de la población en general, que permitió comparar el ajuste psicológico de cada niño o niña y su familia. Los resultados mostraron que el grupo de niños y niñas con cáncer y sus familiares experimentaron significativamente mayor desajuste emocional que los infanyes y sus padres de la comunidad durante el periodo inmediato al diagnóstico. Sin embargo, el número de problemas experimentado por el grupo con diagnóstico de cáncer declinó durante el primer año y se estabilizó a niveles comparables con los encontrados en el grupo de la comunidad en general. Esto les permitió concluir que, aún cuando los resultados son consistentes con reportes en los cuales se sugiere que después de un largo tiempo la prevalencia de problemas psicológicos entre infantes con cáncer es similar a la encontrada en la población en general, también permite comprender mejor el fuerte desajuste emocional que experimentan tanto los niños como sus padres durante el período inmediato al diagnóstico.

Greenberg et al. (1989) encontraron que infantes sobrevivientes de cáncer quiénes reportaron restricciones en sus actividades de la vida diaria mostraron mayores rasgos de depresión respecto a sobrevivientes sin restricciones estando esto en relación directa con el desarrollo psicosocial del niño y la niña. La prevalencia de la depresión fue estudiada usando el inventario de depresión de Beck y el esquema de desórdenes afectivos y de esquizofrenia en una muestra de 30 adolescentes con cáncer. De acuerdo al inventario de Beck, el $87 \%$ de la población estudiada no se encontró deprimida, el 31\% estuvo moderadamente deprimida y ninguno presentó rasgos de depresión severa. De forma similar, en el esquema de desórdenes afectivos y de esquizofrenia los datos no arrojan una historia de depresión en el $75 \%$ de la muestra, más bien se observan rasgos de depresión de menor a mayor en un $10 \%$ de la muestra. Las mujeres puntuaron significativamente más alto que los hombres sobre situaciones que conllevan condiciones físicas no así en los ítems psicológicos. El promedio de respuestas físicas en el Beck fue significativamente mayor que los ítems psicológicos sugiriendo con ello que los síntomas somáticos sobrepasan a los psicológicos en comparación con la norma.

Blum (1992) sugiere que niños y niñas con enfermedades o alteraciones crónicas son depresivos/as no porque ellos tengan en si mismos/as una psicopatología, sino más bien porque ellos se encuentran socialmente mas aislados que sus compañeros/as. También indican que los niños y las niñas enfermos/as llegan a deprimirse cuando ellos no pueden realizar las actividades propias de su edad o cuando sus progenitores les prohíben participar en actividades por sobreprotegerlos. Por su parte Watters y Williamson (1999) concluyen que en el estudio de poblaciones pediátricas los niños y niñas con varios desórdenes, incluyendo dolor crónico, llegan a ser un factor de influencia negativa para una restricción en las actividades diarias, 
es decir, en ocasiones la propia enfermedad física no limita las actividades físicas pero el dolor crónico si, afectando su desarrollo social y conduciendo a la depresión.

Stam, Grootenhuis y Last (2001) hicieron una revisión de las publicaciones realizadas desde 1985 haciendo un análisis de los métodos, instrumentos y muestras utilizadas con respecto a los sobrevivientes de cáncer: su adaptación social y emocional. Concluyen que aunque un gran número de estudios reportan que éstos/as jóvenes se adaptan razonablemente bien, los resultados con respecto a su ajuste emocional y social son muy inconsistentes. La revisión se dividió en cuatro secciones: ajuste emocional, socio-conductual, ajuste de sobrevivientes y finalmente las conclusiones e implicaciones en futuras investigaciones. En forma general establecen que los y las jóvenes sobrevivientes de cáncer no difieren significativamente en su ajuste psicosocial de sus pares. En estudios longitudinales los resultados indican que los/as sobrevivientes tienen más problemas sociales y menos competencia social que sus iguales pero estos problemas tienden a decrecer conforme avanza el tiempo llegando al mismo nivel que el grupo control (Sawyer, et al., 1997, 2000). Se considera importante que en futuras investigaciones se clarifique si todos estos factores se encuentran o no relacionados con el cáncer. Carpentieri (en Stam, 2001) encontró que la competencia social y los problemas de conducta inmediatos al diagnóstico fueron predictores fuertes de desajuste psicosocial en los sobrevivientes.

Otro gran factor es el afrontamiento que hace la familia hacia el cáncer. El funcionamiento de los padres y de la familia influye en el funcionamiento del niño/niña y viceversa. La mayoría de los estudios reportan un adecuado ajuste por parte de la familia pero en algunos casos se establece una sobreprotección o bien mayor rigidez y menor flexibilidad que en los padres con niños y niñas sin cáncer (Greenberg, et al., 1989; Kazak, et al., 1989; Faulkner, Rhonda y Davey, 2002). Otras investigaciones han relacionado la calidad en la comunicación familiar, la cohesión y la adaptabilidad como factores para el éxito psicosocial de los sobrevivientes (Kazak, 1989). Por otro lado se han investigado las habilidades de adaptación social y psicológica en sobrevivientes de cáncer en una muestra de infantes y adolescentes con edades entre los 6 y los 18 años de edad en el momento de la evaluación. Ésta se realizó tanto con los niños y las niñas como con sus padres y maestros/as así como valorando el funcionamiento familiar. Según los resultados no se muestran alteraciones significativas en la adaptación y se concluye que el rol de las dificultades en el aprendizaje y el funcionamiento familiar influye en el ajuste de habilidades sociales en estos/as niños/as y adolescentes (Levin, Brown, Pawletko, Gold, y Whitt, 2000).

En los últimos años la necesidad de valorar la calidad de vida de los adultos sobrevivientes al cáncer infantil se ha enfatizado pudiendo con ello 
determinar la importancia de las secuelas psicológicas a largo plazo que estas personas pueden presentar (Carlson y Bultz, 2003). Langeveld, Stam, Grootenhuis y Last (2002) realizaron una revisión global de los estudios que sobre esta materia se han realizado. El análisis se centra en 30 estudios empíricos publicados desde el año 2001. El abordaje de la calidad de vida se realiza desde diversas dimensiones tales como: funcionamiento físico es decir, salud en general, funcionamiento psicológico, funcionamiento social y funcionamiento sexual. Se considera que los resultados pueden aun no ser muy claros y generalizados sin embargo las primeras conclusiones que se obtienen de estos estudios son: con respecto a salud, la mayoría de los sobrevivientes reporta un adecuado nivel de salud física con excepción de los sobrevivientes de tumores craneales quienes consideran que su funcionamiento físico en general es mas precario, mencionando a la fatiga como un efecto residual del tratamiento. En el funcionamiento psicológico no se hace referencia a fallos en esta área diferentes a sus pares en los grupos control, algunos subgrupos mencionan la presencia de depresión, alteraciones en el humor, tensión, coraje, confusión y ansiedad. Los principales factores de riesgo señalados en estos estudios fueron, el género femenino, mayor edad, presencia de alguna alteración funcional y la irradiación craneal.

En el área social se reporta que los/as sobrevivientes de tumores craneales y de leucemia presentan mayores dificultades. La irradiación craneal y un diagnóstico a temprana edad fueron asociados con un déficit escolar. Un buen grupo de sobrevivientes reportó que su nivel educacional se vio afectado debido a la enfermedad. Para los sobrevivientes mayores, se reporta discriminación en el trabajo y dificultades para obtenerlo aunque esto no es consistente en todos los casos.

Algunos resultados por demás sorprendentes son la existencia de una baja tasa de matrimonios y de paternidad en esta población particularmente en hombres sobrevivientes con diagnóstico de tumor craneal. Ellos reportan que su historia de cáncer infantil, las consecuencias del tratamiento así como los problemas de salud son razones específicas para no contraer matrimonio y por ende para no tener hijos. Muchos sobrevivientes reportan una alta preocupación por su capacidad reproductiva y por las posibles dificultades de salud que sus hijos puedan experimentar en el futuro como resultado de su historia previa de cáncer (Langeveld, et al. 2002).

Este último apartado establece secuelas psicológicas importantes en esta población relacionadas con su autoconcepto, autoconfianza y con la inseguridad y desconfianza con la que miran hacia el futuro, especialmente en cuanto a la prolongación de la vida a través de la procreación de los hijos. Los sobrevivientes estudiados llegan a considerar la posibilidad de volver a vivir su historia médica a través de sus hijos, con lo doloroso y frustrante que 
ello sería o bien con una realidad difícil de asumir como es la esterilidad o dificultades en la capacidad sexual.

Algo que sin duda se puede plantear con certeza, es que no todos los niños/niñas y adolescentes con cáncer sufren de problemas serios de adaptación, algunos son más vulnerables que otros. Es importante entonces identificar los factores que pueden predecir un ajuste psicosocial exitoso. Los resultados al respecto no son muy consistentes y son pocos los factores que están bien definidos. Para algunos autores cuando se tiene una mayor edad al momento de recibir el diagnóstico, un largo tiempo de tratamiento, la terapia de irradiación, y/o algunos efectos posteriores de la medicación se asocian con la presencia de problemas de adaptación.

Como se puede observar, las tendencias de la investigación en general, se han cuestionado el impacto y las secuelas que puede o debe dejar la experiencia estresante del cáncer infantil y su tratamiento, pero a pesar de ello, no se ha podido llegar a una conclusión única acerca de la magnitud de éstas secuelas, principalmente porque no ha existido una uniformidad en los criterios y poblaciones estudiadas. Los resultados deben ser examinados con respecto al tipo de instrumento, la metodología o el grupo control utilizado, o bien, a factores externos que pueden incidir en los resultados tales como el adecuado afrontamiento de la situación por parte de la familia y el apoyo de sus pares.

\section{Conclusiones}

La depresión infantil puede presentarse asociada al diagnóstico y tratamiento de una enfermedad médica crónica o de larga duración como es el caso del cáncer, que puede dejar secuelas psicológicas en sus sobrevivientes.

La edad de aparición de la enfermedad ha sido uno de los factores que se consideran más relevantes para la manifestación de secuelas psicológicas. A menor edad, mayor capacidad de adaptación a los cambios y demandas de la enfermedad, sin embargo, si ésta se manifiesta durante la adolescencia, las secuelas serán mayores debido principalmente al proceso del desarrollo que se está viviendo, esto es, en ésta etapa el/la joven se encuentra en una lucha por su autonomía, independencia y fortalecimiento de su autoconcepto, los que se ven fuertemente dañados por la aparición del cáncer. La autoimagen y la relación de igual con sus pares se encuentran debilitadas, las secuelas suelen ser más graves en los casos en los que el autoconcepto es más pobre, aunado a rasgos depresivos. Pero también es importante resaltar que es en la adolescencia en donde el proceso de rebeldía es más manifiesto y con ello la 
lucha del joven por superar y vencer la enfermedad permitiéndole en la mayoría de los casos salir airoso de la situación.

También juega un papel importante el proceso de adaptación a la enfermedad con sus restricciones y exigencias. Muchas de las manifestaciones afectivas que aparecen, principalmente los estados depresivos, son resultado no de la enfermedad en sí, sino de sus consecuencias, tales como, las restricciones en actividades físicas o de juego; la imposición de un régimen alimenticio o de medicamento; el cambio de autoimagen debido a la pérdida de pelo o de peso corporal; la pérdida de la rutina diaria como la asistencia a clase o la convivencia con los compañeros debido a las constantes visitas médicas u hospitalizaciones.

Existen momentos críticos que evidencian con mayor fuerza alteraciones psicológicas y éstos suelen ser tanto el momento inmediato al diagnóstico como el proceso mismo del tratamiento. Cuando se recibe el diagnóstico el niño-joven (la niña/joven) requiere entender, aceptar, lo que le está ocurriendo y los cambios que esta enfermedad le ocasionarán, por lo cual no es de extrañar que en éstos momentos la depresión aparezca con intensidad. De igual forma, suele ocurrir cuando se inicia el tratamiento, las consecuencias que éste trae, como las de carácter físico que suelen ser peores y más aversivas que la propia enfermedad, sumiendo a la persona en un mundo de dolor, contradicción y aflicción. El seguimiento del tratamiento y las recaídas se acompañan por lo general de síntomas depresivos y ansiosos, sin embargo, finalizado éste, continua un periodo largo de sobrevivencia y curación que suele ir acompañado de un optimismo generalizado a pesar de la presencia de una cierta incertidumbre y vulnerabilidad.

De los estudios revisados se desprende que para un grupo numeroso de autores los niños y las niñas con cáncer logran una adecuada adaptación psicosocial con mínimos problemas específicos. Sin embargo, es menester resaltar que aunque la mayoría de los sobrevivientes al cáncer infantil parecen no presentar secuelas psicológicas importantes, no se puede dejar de lado la experiencia negativa y confrontativa vivida durante el proceso de una enfermedad y un tratamiento, largo, penoso, invasivo y restrictivo.

Esta experiencia, sin duda ha dejado huellas que se verán reflejadas en acciones futuras importantes en la vida de ésta persona, tales como la manifestación de sus propias creencias y estilos de afrontamiento que estos sobrevivientes se han formado a lo largo de su niñez y adolescencia y se hacen evidentes en sus planes a futuro.

Algunos de los principales temores reportados por los sobrevivientes de cáncer son la incertidumbre y el miedo con respecto a su capacidad sexual, reproductiva y al temor de ser ellos los responsables de transmitir el cáncer a sus hijos/as e inclusive prevalece en la mayoría de ellos el fantasma de la enfermedad quien puede regresar en cualquier momento y lo que es peor hacerse presente en sus hijos/as. 
Un buen grupo de sobrevivientes de cáncer han mostrado una mayor capacidad de recuperación y éxito en su reajuste psicológico tanto en el tratamiento como a la enfermedad, pero a pesar de las pruebas de adaptación exitosas, una parte de los estudios señalan evidencias sobre un importante subgrupo de pacientes sobrevivientes que experimentan dificultades psicológicas. Es necesario entonces, que durante un periodo mayor, los sobrevivientes de cáncer sean apoyados para que continúen su desarrollo social, emocional y cognitivo el cual les permita hacer ajustes psicológicos por largo tiempo y junto con ellos su familia.

\section{Referencias}

Allen, R., Newman, S. \& Souhani, R.L. (1997). Anxiety and depression in adolescent cancer: Findings in patients at the time of diagnosis. European Journal of Cancer, 33, 1250-1255.

Archenbach, T. M., (Ed.). (1983). Manual for the child behavior. Checklist and revised child behavior profile. Vermont, United States of America.

American Psychiatric Association. (1994). Diagnostic and Statistical Manual of Mental Disorders (4th ed.). Washington, EE.UU.

Augelino, A. F. \& Treisman, G. J. (2001). Major depression and demoralization in cancer patients: Diagnostic and treatment considerations. Support Care Cancer 9, 344-349.

Balen, R., Fielding, D. \& Lewis, I. J. (1998). An activity week for children with cancer: Who wants to go and why?. Child Care Health, 24 (2), 169-177.

Bauld, C., Anderson, V. \& Arnold, J. (1998). Psychosocial aspects of adolescent cancer survival. Journal of Pediatric and Child Health, 34, 120-126.

Barley, J. B. \& Mosher, R. B. (1997). Adjustment responses of children and their mothers to cancer: Self-care and anxiety. Oncology Nursing Forum, 2, 519-525.

Benedito, M. C., López, J. A., Serra, J., Harto, M., Gisbert, J., Mulas, F. \& Freís, T. (2000). Secuelas psicológicas en los sobrevivientes a largo plazo de cáncer. Anales Españoles de Pediatría, 53 (6), 553-560.

Bennett, D. S. (1994). Depression among children with chronic medical problems: A metaanalysis. Journal of Pediatric Psychology, 19, 149-169.

Birmaher, B. \& Brent D. (1998). Practice parameters for the assessment and treatment of children and adolescents with depressive disorders. Journal of the American Academy of Child Adolescence Psychiatry, 37, 63-83.

Block, S. D. (2000) Assessing and managing depression in the terminally ill patient. ACPASIM End of-Life Care Consensus Panel. Annals of Internal Medicine, 132 (3), 209-218.

Blum, R.W. (1992). Chronic illness and disability in adolescence. Journal of Adolescence Health, $13,364-368$.

Boeving, A. (2000). Adjustment to cbildhood chronic illness: prediction of psychological adjustment with an investigation into spiritual coping. Doctoral dissertation in psychology, Virginia Polytechnic Institute and State University. Blacksburg, Virginia, United States.

Bottomley, A. (1998). Depression in cancer patients: A literature review. European Journal of Cancer Care, 7, 181-191.

Bukberg, J., Penman, D. \& Holland, J. (1984). Depression in hospitalized cancer patients. Psychosomatic Medicine, 46 (3), 199-212.

Canning, E. H., Hanser, S. B., Shade, K. A. \& Boyce, W. T. (1992). Mental disorders in chronically ill children: Parent-child discrepancy and physician identification. Pediatrics, 90, 692-696.

Carlson, L. \& Bultz, B. (2003). Benefits of psychosocial oncology care: Improved quality of life and medical cost offset. Health and Quality of Life Outcomes, 1-8. 
Clay, A. R. (1999). Psychologists help prevent and manage learning difficulties brought on by cancer treatments. American Psychological Association, Monitor Online, 30 (6). Recuperado el 15 de agosto de 2003, de http://www.apa.org/monitor/jun99/tools.html

Del Barrio, V. \& Moreno C. (1996). Evaluación de la depresión y la ansiedad infantil. Ansiedad $y$ estrés, 2 (2-3), 131-158.

Del Barrio, V. (1997). La depresión infantil. Causas, evaluación y tratamiento. Madrid: Ariel.

Del Barrio, V. (2000a). La depresión infantil. Factores de riesgo y posibles soluciones. Madrid: Aljibe.

Del Barrio, V. (2000b). Pobre niño rico. La depresión infantil, una enfermedad de los países desarrollados. España: Aljibe.

Del Barrio, V. (2001) Avances en depresión infantil y juvenil. Información psicológica. Dossier. 323.

Depression PDQ. (s/f). National Cancer Institute. Recuperado el 16 de junio de 2003 de http://cancer.gov/cancer information/coping.

Derogatis, L. R., Morrow G. R. \& Fettings, J. (1983). The prevalence of psychiatric disorders among cancer patients. Journal of American Medical Academy, 249 (6), 751-757.

Deuber, C. M. (1982). Depression in the school-aged child: Implications for primary care. Nurse Practitioner, 7 (8), 26-30.

Donnelly, S. \& Walsh D. (1995). The symptoms of advanced cancer. Seminars in Oncology, 22 (13), 67-72.

Druss, B. J, Rosenbeck, R. A. \& Sledge, W. H. (2000). Health and disability cost of depressive illness in a major U.S. corporation. American Journal of Psychiatry, 157, 1274-1278.

Essau, C. A. \& Petermann U. (1995). Depression in children and adolescents. Psychology, Psychopathology, Psychotherapy, 43 (1), 18-33.

Faulkner, A. Rhonda, A. \& Davey, M. (2002). Children and adolescents of cancer patients: The impact of cancer on the family. American Journal of Family Therapy, 30, 63-72.

Frank, N. C., Blount, R. L. \& Brown, R. T. (1997). Atributions, coping and adjustment in children with cancer. Journal of Pediatric Psychology, 22, 563-576.

Fresneda, C. (2003). 300,000 niños menores de doce años toman Prozac en Estados Unidos, un país donde el 25\% de los menores sufre depresión. El Mundo. Recuperado el 16 de junio de 2003 de: http://www.lafogata.org/003inter/inter1/listado.htm

Fritz, G. K., Williams, J. R. \& Amylon, M. (1988). After treatment ends: Psychosocial sequels in pediatric cancer survivors. American Journal of Orthopsychiatry, 58 (4), 552-561.

Grau, R. C. \& Cañete N. A. (2000). Niños con cáncer. Vivir...su juego más divertido. España: ASPANION.

Greenberg, H. S., Kazak, A. E. \& Meadows, A. T. (1989). Psychology functioning in 8 to 16 year old cancer survivors and their parents. Journal of Pediatric, 114 (3), 488-493.

Henriksson, M. M., Isometsä, E. T. \& Hietanen P. S. (1995). Mental disorders in cancer suicides. Journal of Affective Disorders, 36 (1-2), 11-20.

Kaplan, S. L., Busner, J., Weinhold, C. \& Lenon P. (1987). Depressive symptoms in children and adolescents with cancer: A longitudinal study. Journal of the American Academy of Children \& Adolescence Psycbiatry, 26 (5), 782-787.

Kashani, J. \& Hakami, N. (1982). Depression in children and adolescents with malignancy. Canadian Journal of Psycbiatry, 27 (6), 474-477.

Kazak, A.E. (1989). Psychological issues in childhood cancer survivors. Journal of Pediatric Oncology Nursing, 6 (1), 15-16.

Kazak, A. E. \& Meadows, A. T. (1989). Families of young adolescents who have survived cancer: Social-emotional adjustment, adaptability, and social support. Journal of Pediatric Psychology, 14, 175-191.

Koocher, G., O’Malley, J., Gogan, J. \&Foster, D. (1980). Psychological adjustment among pediatric cancer survivors. Journal Cbild Psycbol Psychiatry, 2, 165-173.

Langeveld, N. E., Stam, H., Grootenhuis, M. A. \& Last, B. F. (2002). Quality of life in young adult survivors of childhood cancer. Support Care Cancer, 10, 579-600.

Lebaron, S. y Zeltzer, L. K. (1984). Assessment of pain and anxiety in children and adolescents by self-reports, and a behavior checklist. Journal of Consulting \& Clinical Psychology, 52, 729-738.

Levin, N., Brown, R. Pawletko, T., Gold, S. \& Whitt, J. (2000). Social skills and psychological adjustment of child and adolescent cancer survivors. Psychooncology, 9 (2), 113-126.

Actualidades en Psicología,

20, 2006, 22-44 
Lloyd Williams, M. \& Friedman, T. (2001). Depression in palliative care patiens-a prospective study. European Journal of Cancer Care, 10, (4), 270-274.

Lynch, M. E. (1995). The assessment and prevalence of affective disorders in advanced cancer. International Journal of Palliative Care, 11 (1), 10-18.

Links, P. S. \& Stockwell, M. L. (1985). Obstacles in the prevention of psychological sequeals in survivors of childhood cancer. American Journal of Pediatric Hematolology/Oncology, 7 (2), 132-140.

Malmquist, C. O. (1983). Major depression in childhood: Why don't we know more? American Journal of Orthopsychiatry, 53 (2), 262-68.

Masi, G., Favilla, L., Mucci, M. \& Millepiedi, S. (2000). Depressive comorbidity in children and adolescents with generalizated anxiety disorder. Child Psychiatry and the Human Development, 30 (3), 205-215.

Massie, M. J. \& Holland, J. C. (1987). The cancer patient with pain: Psychiatric complications and their management. Medical Clinic North American 71 (2), 243-58.

McDaniel, J. S., Musselman, D.L., Porter, M. R., Reed, D. A. \& Nemeroff, C. B. (1995). Depression in patients with cancer. Diagnosis, biology and treatment. Archive General Psychiatry, 52 (2), 89-99.

Meyer, T. J. \& Mark, M. M. (1995). Effects of psychosocial interventions with adult cancer patients: A meta-analysis of randomized experiments. Health Psychology, 14 (2), 101-08.

Moe, P.J. \& Holen, A. (2000). Long-term coping in childhood cancer survivors-influence of illness, treatment and demographic factors. Acta Pediatric, 89 (1), 4-6.

Mulhern, R. K., Fairclough, D. I., Smith, D. \& Douglas, S. M. (1992). Maternal depression: Assessment methods and physical symptoms affect estimates of depressive symptomatology among children with cancer. Journal of Pediatrics Psychology, 17, 313-326.

Naughton M. \& Homsi J. (2002). Symptom assessment in cancer patients. Current Oncology Reports, 4, 256-263.

Nerville, K. (1996). Psychological distress in adolescents with cancer. Journal of Pediatric Nursing, 11, 243-251.

Nissen, G. (1987). La depresión en la niñez y en la adolescencia: diagnóstico y tratamiento. Psicopatología, 7 (3), 303-314.

Noll, B., Bukowski, W. M., LeRoy, S. S., Rogosch, F. A. \& Kulkarni, R. (1991). Peer relationships and adjustment of children with cancer. Journal Pediatric Psychology, 16, 307-327.

Noll, B., Gartstein, A., Vannatta, K., Correll, B., William M. \& Davies, H. (1999). Social, emotional and behavioral functioning of children with cancer. Pediatrics, 103 (1), 71-79.

O’Malley, J. E., Koocher, G. \& Foster, D. (1979). Psychiatric sequels of surviving childhood cancer. American Journal of Orthopsychiatry, 49 (4), 608-16.

Pauchard, H. (2002). La depresión infantil: sintomas y tratamiento. Recuperado el 17 de Julio de 2003. de http://www.relaciones-humanas.net/depresioninfantil.html.

Perrone, J. (1993). Adolescents with cancer are they at risk for suicide?. Journal of Pediatric Nursing 19 (1), 22-25.

Petersen, A. C., Compas, B. E., Brooks-Gunn, J., Stemmler, M., Ey, S. \& Grant, K. E. (1993). Depression in adolescences. American Psychologist, 48, 155-168.

Petersen, R. W. \& Quintivan, J.A. (2002). Preventing anxiety and depression in gynecological cancer: A randomize controlled trial. British Journal of Obstetrics and Gynaecology 109 (4), 386-394.

Peveler, R., Carson, A. \& Rodin, G. (2002). Depression in medical patients. ABC of psychological medicine. British Medical Journal, 325, 149-152.

Rechner, M. (1990). Adolescentes with cancer: Getting on with life. Journal of Pediatric and Oncology Nursing, 7 (4), 139-144.

Ressel, W. (2003). NIH Releases statement on managing pain, depression, and fatigue in cancer. American Family Psychsician, 67 (2), 423-425.

Ringdal, G. L. (1995). Correlates of hopelessness in cancer patients. Journal of Psychosocial Oncology, 13 (3), 47-66.

Sawyer, M., Antoniou G., Nigoyen A., Tooogood I. \& Rice, M. (1995). A prospective study of the psychological adjustment of children with cancer. American Journal of Pediatric Hematology/Oncology, 17, 39-45. 
Sawyer, M., Antoniou G., Tooogood I., \& Rice, M. (1997). Childhood cancer: A two-year prospective study of the psychological adjustment of children and parents. Journal of the American Academy of Children \& Adolescent Psychiatry, 36 (12), 1736-1743.

Sawyer, M., Antoniou G., Tooogood I., Rice, M. \& Baghurst P. (2000). Childhood cancer: A 4-year prospective study of the psychological adjustment of children and parents. Journal Pediatric of Hematology Oncology, 22, 214-220.

Sean, P. (2002). Children adapt to chronic illnesses. Mental Health Weekly. Recuperado el 17 de Julio de 2003 de http://www.findarticles.com

Shemesh, E., Bartell, A. \& Newcorn, J. (2002). Assessment and treatment of depression in medically III children. Current Psychiatry Reports, 4, 88-92.

Stam, H., Grootenhuis, M. A. \& Last, B.F. (2001). Social and emotional adjustment in young survivors of childhood cancer. Support Care Cancer 9, 489-513.

Stein, D., Witztum, E. \& De-Nour, A. K. (1989). Adolescent attitudes toward suicide. Israel Journal of Psychiatry and Related Sciences, 26 (1-2), 58-68.

Tebbi, C., Bromberg, C. \& Mallon, J. C. (1988). Self-reported depression in adolescent cancer patients. American Journal of Pediatric Hematology/Oncology, 10 (3), 185-190.

Von E., Enskär, K., Kreuger, A. \& Sjödén, P. (2000). Self-esteem, depression and anxiety among swedish children and adolescents on and off cancer treatment. Acta Pediatric, 89, 229-236.

Watson, M., Haviland, J. S., Greer, S., Davidson, J. \& Bliss, J. M. (1999). Influence of psychological response on survival in breast cancer: As population-based cohort study. Lancet, 354, 1331-1336.

Watson, M., Haviland, J., Greer, S., Davidson, J. \& Bliss, J. (2002). Quality of life in childhood cancer survivors. Psychooncology, 11 (2), 132-141.

Watters, A. \& Williamson, G. (1999). The role of activity restriction in the association between pain and depression: A study of pediatric patients with chronic pain. Children's Health Care, 28, (1), 33-51.

White, C. A. \& Macleod, U. (2002). Cancer. ABC of psychological medicine. British Medical Journal. 325, 377-380.

Wilkinson, S. \& Kitzinger, C. (2000). Thinking differently about thinking positive: A discursive approach to cancer patients' talk. Society Scientific Medical, 50 (6), 797-811.

Woffe, J., Grier H. \& Klar, N. (2000). Symptoms and suffering at the end of life in children with cancer. New England Jounal of Medicine, 342, 326-333.

Worchel, F., Nolan, B., Willson, V., Purser, J., Copelan, D. \& Pfefferbaum, B. (1988). Assessment of depression in children with cancer. Journal of Pediatric Psychology, 13, 101112.

Recibido: 24 de febrero de 2004

Aprobado: 29 de agosto de 2004

Actualidades en Psicología,

$20,2006,22-44$ 